\title{
Research on Aerodynamic Noise Reduction of Wind Turbine Blades Based on Tyto longimembris Bionic Tail Edge
}

\author{
Shaoyu Wang ${ }^{1, \text { a }}$ and Quan Wang ${ }^{1}$ \\ ${ }^{1}$ North China Electric Power University, Beijing, 102206 \\ aalanncepu@foxmail.com
}

Keywords: Bionic tail edge; DU300 airfoil; Aerodynamic noise; LES turbulence model

\begin{abstract}
In this paper, CFD software is used to simulate the Fluent numerical simulation of the DU300 airfoil trailing edge and the trailing edge of DU300. Using LES turbulence model, calculating the FW-H equation, the noise transformed by FFT transform, and the sound field distribution of the blade aerodynamic noise is obtained, and the variation of the aerodynamic noise of the monitoring points at different locations and the attenuation law are analyzed and compared. After DU300 airfoil blunt trailing edge increasing Bionic trailing edge, the influence of aerodynamic performance of the airfoil is very small, and it has a significant effect on the noise reduction. Which provides a certain reference value for the optimization design of wind turbine airfoil, the sound field distribution of the bionic trailing edge has certain broadband characteristics.
\end{abstract}

\section{Introduction}

The noise of the wind turbine generator is largely derived from the aerodynamic noise. The aerodynamic noise is more complex, and it is closely related to the complex flow around the blade.in existing research, Li Dian found that owl class with unique wings airfoil and the structure of feathers has great reference value on the blade design and can effectively improve the blade aerodynamic performance and acoustic properties. So this paper starting from the perspective of engineering bionics, through in-depth study of the mechanism of the Grass Owl silent flight, numerical simulation and prediction of aerodynamic noise, provides a new working principle and design idea for the noise reduction technology of wind power generation.

At present, the research on the trailing edge is mainly about the serrated trailing edge. Xue Weicheng on the noise reduction experiment of the serrated trailing edge, found that comparing the Airfoil with serrated trailing edge with the original airfoil, the effect of the medium low frequency and high frequency noise reduction is obvious(over $2 \mathrm{db}$ ). This paper will be more accurate simulation of the bionic Grass Owl wing trailing edge, by getting the features of Grass Owl wings feathers trailing edge wavy edge extraction to establish the trailing edge of the wave shaped blade and explore its noise reduction performance.

\section{The Parameters Determination of Grass Owl Imitation Leaf Tail Edge}

Deng Lei and other studied the aerodynamic performance of the blunt large thickness trailing edge and found that this type of airfoil has good aerodynamic and structural advantages which can make a part of the pressure recovery occurred in the wake and slow the trend of boundary layer separation, so the bionic trailing edge of this paper will be designed on the basis of DU-300 blunt trailing edge airfoil.

This paper is drawing the 2D feature extraction Grass Owl wings referring to Liao Geng hua Method of measurement of the long eared owl wing morphology. Imitating the Grass Owl gliding wing shape expanded naturally, after completely expanding Grass Owl wings of nature death, Getting the wings fully expanded photos with the help of a high pixel digital camera and after treatment, Grass Owl wing of the two-dimensional shape image are shown in Fig. 1. 


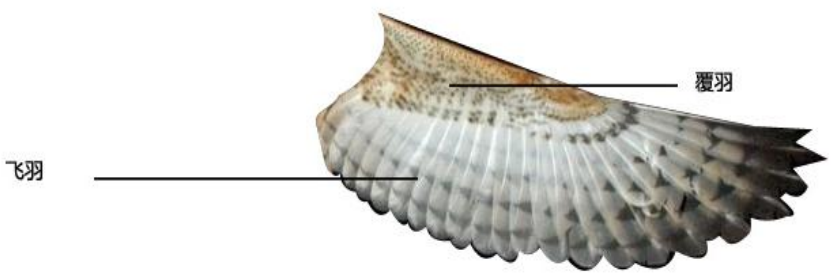

Figure 1-1

This paper is mainly for the trailing edge of blade for biomimetic treatment, so focus on the initial wave type Grass Owl feather structure trailing edge parameter measurement. Among them, the wavy structure of the initial trailing edge is mainly expressed by the wave spacing $S$, the wave height $h$ and the wave width $\mathrm{W}$. The measurement results are shown in Table 1. According to the measurement results of the trailing edge of Grass Owl wing feather, the trailing edge of the wind turbine blade is shown in Fig. 1-1, and its specific parameters are shown in Table 1.

Table 1

\begin{tabular}{|c|c|c|c|}
\hline Structure parameter & $\mathrm{s} / \mathrm{mm}$ & $\mathrm{h} / \mathrm{mm}$ & $\mathrm{w} / \mathrm{mm}$ \\
\hline Grass Owl feather at trailing edge & $20-38$ & $20-55$ & $18-33$ \\
\hline Imitation leaf trailing edge & 93 & 65 & 90 \\
\hline
\end{tabular}

\section{Numerical Calculation of Bionic Blade}

Grid Division and Boundary Conditions. Building bionic blade model in ANSYS ICEM CFD, the mode is introduced into fluent 16 to carry out the blade geometry trimming and calculation domain mesh. Using hybrid grid, trailing edge section to generate structured mesh, Hybrid grid, with the rest of the unstructured grid, outfield uses: C-block, also of the airfoil model in the vicinity of the mesh refinement encryption processing, between the computational domain and the outer domain with interface connected. The final mesh is shown as the extra grid, and the total number is 1 million 700 thousand. In order to ensure the validity of the results of grid computing, grid does independence verification. Combined with the best operating point of the airfoil, Setting import speed to $43.8 \mathrm{~m} / \mathrm{s}$, the angle of attack of 4 degrees,

Outlet pressure of $0.1 \mathrm{MPa}$, no slip wall boundary conditions are adopted for Reynolds number bionic airfoil wall surfaces, and the length of the chord Reynolds number is about 3 million. In order to improve the computational efficiency, the calculation of flow field is carried out the steady state calculation by using SA model, after convergence, the LES model is used to the transient calculate.

Numerical Calculation Model. In this paper, large eddy simulation is used to the steady state calculation. The basic assumption of LES is that energy, momentum, mass, and other scalar quantity which are mainly transported by large eddy. The geometry and boundary conditions of fluid determine the characteristics of large eddy, and the flow characteristics are mainly reflected in the large vortex. Small scale vortex affected by the geometrical boundary condition is small and is isotropic. The governing equations of LES are obtained by filtering the $\mathrm{N}-\mathrm{S}$ equation in the wave number space or physical space. The filtering process is to remove the vortex which is smaller than the width of the filter or given the physical width, so that the control equation of the large vortex can be obtained.

$$
\begin{gathered}
\frac{\partial \rho}{\partial \mathrm{t}}+\frac{\partial \rho u_{i}}{\partial x_{i}}=0 \\
\frac{\partial\left(\rho \overline{u_{i}}\right)}{\partial \mathrm{t}}+\frac{\partial\left(\rho \overline{u_{i}} \bar{u}_{j}\right)}{\partial x_{j}}=\frac{\partial}{\partial x_{j}}\left(\mu \frac{\partial \overline{\sigma_{i j}}}{\partial x_{j}}\right)-\frac{\partial \bar{p}}{\partial x_{i}}-\frac{\partial \tau_{i j}}{\partial x_{j}}
\end{gathered}
$$


Among them, $\sigma_{i j}$ is the stress tensor, $\tau_{i j}$ for the sub grid stress

\section{Analysis of the Calculation Results}

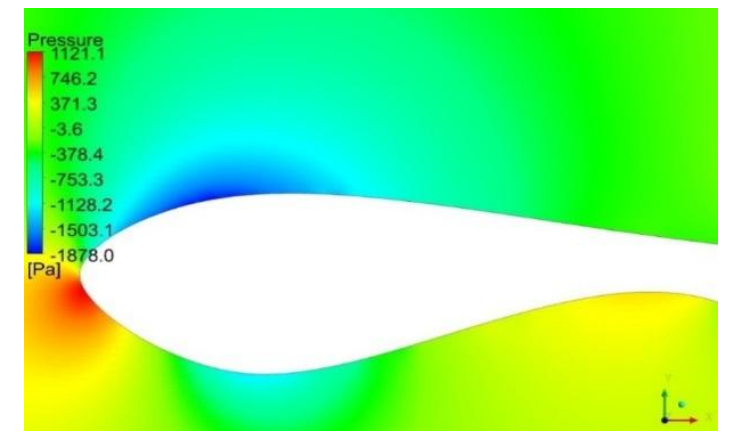

(a)Blunt trailing edge airfoil section

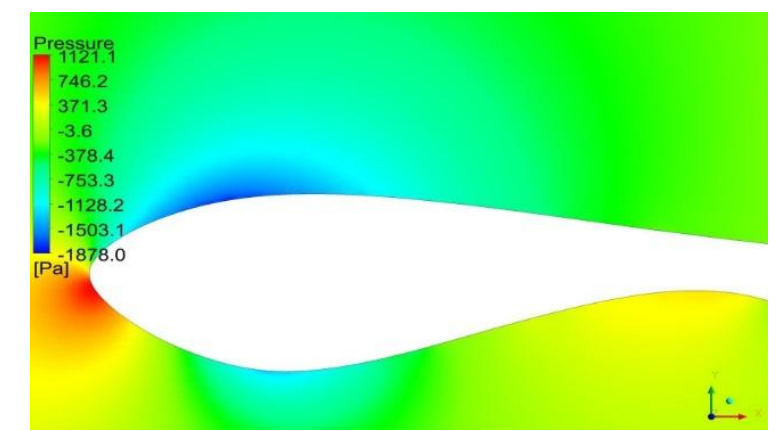

(b) Bionic trailing edge airfoil section

Figure 3-1. Picture of pressure cloud near the blade when $\mathrm{z}=0.1 \mathrm{~m}$ ross-section $\alpha=4^{\circ}$,

Pressure Distribution around an Airfoil. seeing from the pressure cloud image, at the 4 degree angle of attack, the $\mathrm{Z}=0.1 \mathrm{~m}$ cross section, the maximum static pressure appears near the leading edge of the airfoil, and at the leading edge of the airfoil section. The pressure gradient changes drastically. At the same time, on the suction surface, the negative pressure area increased, due to the influence of the angle of attack, the pressure of the two kinds of airfoil is asymmetrical. But by comparing the pressure distribution of the trailing edge airfoil section and the trailing edge airfoil section, we can find that the different trailing edge shapes have little influence on the pressure distribution of the entire surface, which is almost no change.

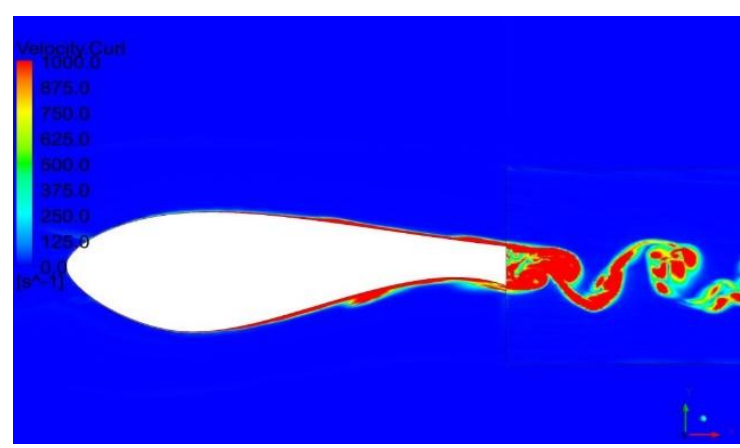

(a) Blunt trailing edge

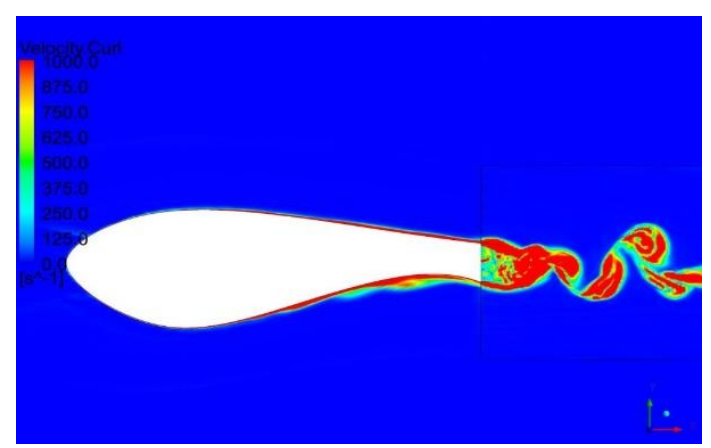

(b) Bionic trailing edge

Figure 3-2. Eddy currents in the vicinity image of the $\mathrm{z}=0.1 \mathrm{~m}$ cross section of $\mathrm{a}=4^{\circ}$

What can be seen from the eddy current diagram, in the 4 degree angle of attack, the $Z=0.1 \mathrm{~m}$ section is that, The trailing edge of the blunt trailing edge airfoil is separated from the air flow, and the flow separation is obvious and Periodic vortex shedding induced by pressure pulsation in the fluid, resulting in larger noise. After the airfoil section is equipped with bionic trailing edge, there is still an airflow separation phenomenon, and there are two distinct shedding vortexes, but the separation point of the detached vortex is delayed. At the same time, due to the installation of bionic trailing edge, then the vortex is broken down into a number of small vortexes, slows down the pressure pulsation. By comparing the vortex distribution images of the trailing edge and the trailing edge section, the vortex distribution of the trailing edge section, we can find that, the vortex distribution in the tail section of the airfoil with bionic trailing edge is more complex, and the original vortex is broken into small vortex, and the small eddies have a certain effect on the numerical value of sound pressure level. 
Influence of the Bionic Trailing Edge on the Noise Field. Using LES turbulence model carries out the noise sound field numerical simulation of DU300 blunt trailing edge airfoil section, the installation of bionic trailing edge section. According to the result of noise calculation, analyzed and compared the receiving point and wake noise sound pressure distribution in two cases. Taking the axial midpoint of airfoil as the coordinate origin, establish the coordinates of the monitoring point, around the airfoil layout two laps of the monitoring points, the radius of $10 \mathrm{C}$ and $15 \mathrm{C}$.

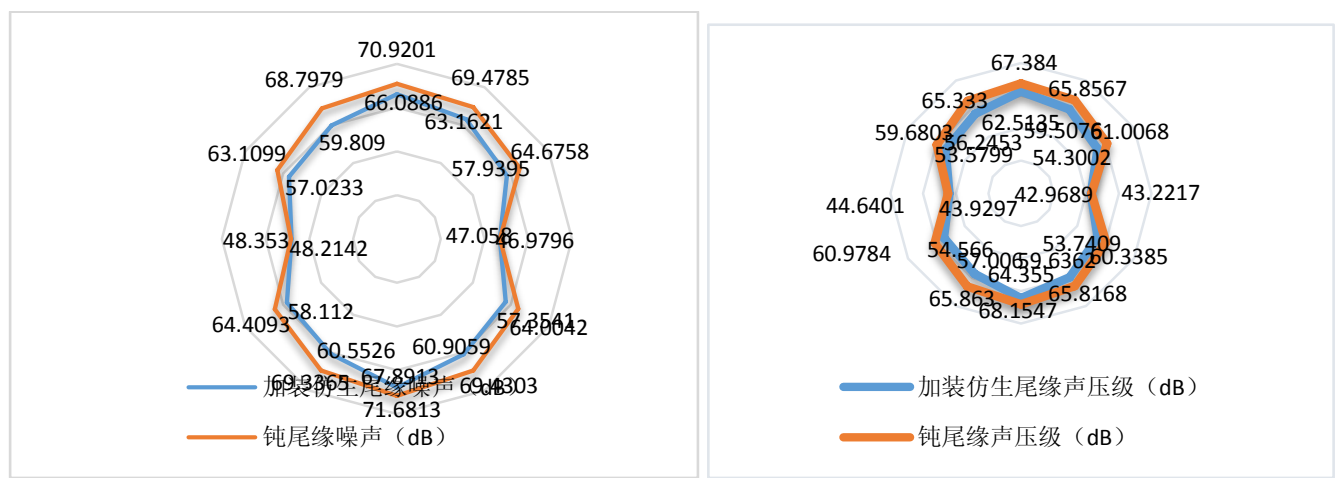

Figure 3-3 monitoring radius of sound pressure level for 10C data

Figure 3-4 monitoring radius of sound pressure level for $15 \mathrm{C}$ data

What can be seen from the Fig. 3-3 and Fig. 3-4 are that, for a single airfoil, Position of monitoring points above the suction surface

Due to the separation of sound pressure level, generated vortex, resulted in the increase of turbulence intensity, fluctuating pressure increases, so the value is slightly more, and compared to the number of two monitoring points in the axial direction and the other monitoring sites, the sound pressure level numerical is much smaller. Secondly, after numerical comparison with two kinds of airfoil section SPL, we can get that the noise reduction effect is not obvious in the axial direction of the airfoil section with bionic trailing edge, but in other direction, the noise is reduced by at least $5 \mathrm{~dB}$, which indicates that the bionic trailing edge has a certain effect on noise reduction. And comparison of different monitoring radius of sound pressure level data, It can be found that with the increase of the distance, the sound pressure level becomes smaller and smaller, and the radiation of the noise is spread to the surrounding radiation, in areas with strong pressure pulsation sound pressure level value is greater.

Two monitoring points were selected from all monitoring points to study the noise frequency distribution.
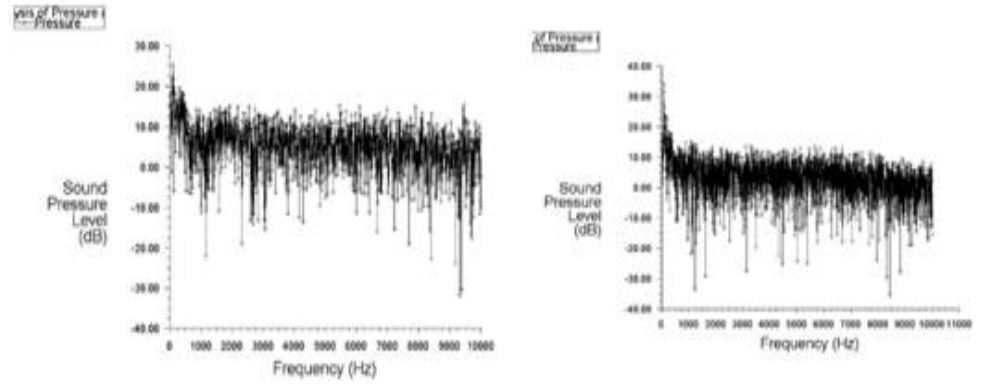

(a)Monitoring point 10
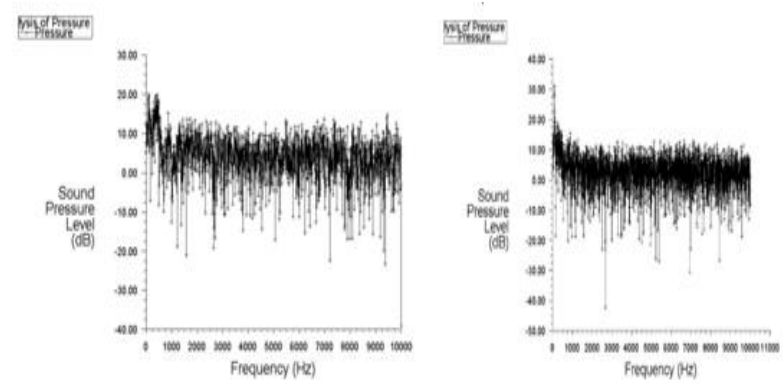

(b) Monitoring point4

Figure 3-5. The Les model of 4 degrees of noise sound pressure level data

Fig. 3-5 is the SPL-f distribution curve of the DU300 blunt trailing edge airfoil section under the sound pressure level with the frequency of the noise monitoring point 4 and 10 at the 4 degree angle of attack. The SPL-f distribution curve of sound pressure level along with the frequency of the bionic trailing edge airfoil is on the left side, the SPL-f distribution curve of sound pressure level with the frequency of the airfoil section of the usual blunt trailing edge being the right. Monitoring 
point 4 , monitoring point 10 are located at the front edge and trailing edge of the airfoil two monitoring points, through the analysis of these monitoring points of the sound pressure level with the frequency of the SPL-f distribution curve, to find the distribution characteristics of two kinds of airfoil noise.

Analyze Fig. 3-5. Sound pressure level data, at the airfoil leading edge of the monitoring point 10 , both in the low frequency band has obvious dominant frequency. With the increase of frequency, the frequency spectrum shows broadband characteristic, but the peak value of the two is different. The peak value was obviously decreased after the bionic trailing edge, but the leading edge noise was not related to the effect of the separation of the tail fluid. So from Fig. 3-3, Fig. 3-4, we can find that, whether the installation of bionic trailing edge on the front edge of the noise reduction effect. By contrast tail margin monitoring point 4 , the former low frequency has no obvious main frequency, and the later low frequency come out peak value, overall sound pressure level numerical and compared to the original drop a lot of so that noise reduction measures have certain effect. After $4000 \mathrm{~Hz}$ mainly reflecting the broadband characteristics of noise, the main characteristic is that the energy of high frequency position frequency is basically the same, the frequency band is wider, and the amplitude is basically unchanged. This is due to the high degree of instability of the flow around the airfoil, which leads to the trailing edge part of the vortex structure, which makes the noise present broadband characteristics.

\section{References:}

[1] Li Dian Liu Xiaomin, research of the aerodynamic performance For the typical imitation owl wing airfoils blade fan design [J].Fan technology, 2016 (01): 22-29.

[2] Xue Weicheng. Experimental study on the noise reduction of the trailing edge airfoil [D].Institute of engineering thermal physics, Chinese Academy of Sciences, 2015

[3] Derrick Custodio. The effect of humpback whale-like leading edge protuberances on hydrofoil performace [D]. Worcester Polytechnic Institute, 2007.

[4] Deng Lei,Qiao zhide, Yang Xudong, Xiong Juntao. Study on aerodynamic performance of large thickness blunt trailing edge airfoil [J]. Aviation computing technology, 2011, 41 (2): 43-47.

[5] Liao Genghua. The research on long eared owl wing aerodynamic and acoustic characteristics and application of bionic [D]. Jilin University, 2013.

[6] Fan Yanhong, Song Wenping, Wang Yue. Research on large eddy simulation of wind flow around a wind turbine airfoil [J]. Journal of solar energy, 2015, 36 (6): 1423-1428.

[7] Powell A. Theory of vortex sound [J]. Acoust.Soc. 1964, 36(8): 177-195.

[8] Farassat F, Myers M K. Extension of Kirchhoff's Formulation to Radiation form Moving Surface. Journal of sound and vibration [J], 1988, 123(3): 451-460.

[9] Di Francescantonio P. A New Boundary integral Formulation for the Prediction of Sound Radiation [J], Journal of sound and vibration, 1997, 202(4): 191-509.

[10] Singer B A, Brentner K S, Lockard D P. Simulation of acoustics scatting from a trailing edge[J]. Journal of sound and vibration, 1999, 230(3): 544-560. 